\title{
Effective Aperture of Antenna Directivity Bounding and Its Radiation Aperture
}

\author{
MD. Javeed Ahammed ${ }^{1}$, Dr. R.P. Singh ${ }^{2}$, Dr. M. Satya Sai Ram ${ }^{3}$ \\ 1.Research Scholar, ECE Department, Sri Satya Sai University of Technology and Medical Science, \\ Sehore, Bhopal, Madhya Pradesh, India. E-mail: ahammed.javeed@gmail.com \\ 2. Vice- Chancellor, Sri Satya Sai University of Technology and Medical Science, Sehore, Bhopal, \\ Madhya Pradesh, India. \\ 3. HOD, ECE, Chalapathi Institute of Engineering and Technology, Chalapathi Nagar, Guntur, \\ Andhra Pradesh, India.
}

\begin{abstract}
In this paper, among the earliest type of antennas in production was aperture type. These antennas were rigid and consisted of a parabolic, paraboloidal, cylindrical, or spherical shape. A major limitation of this type of antenna stems from the fact they could only give one particular radiation pattern, and if one wanted to scan the signal from one point to another, then the whole structure had to be moved which meant the satellite had to be realigned. This major shortcoming led to the development of the more costly phased array technology and other technologies where beam scanning was exploited. The aperture is defined as the area, oriented perpendicular to the direction of an incoming radio wave, which would intercept the same amount of power from that wave as is produced by the antenna receiving it. At any point, a beam of radio waves has an irradiance or power flux density which is the amount of radio power passing through a unit area of one square meter.
\end{abstract}

KEYWORDS: Power Flux Density (PFD), Reflector aperture, Shape Memory Alloys (SMA), Poly Vinyli Dene Fluoride $(P V D F)$, radiation lobe $(R L)$.

\section{I.INTRODUCTION}

Antennas are key components of any wireless system. An antenna is a device that transmits and/or receives electromagnetic waves. Most antennas are resonant devices, which operate efficiently over a relatively narrow frequency band. An antenna must be tuned to the same frequency band that the radio system to which it is connected operates in, otherwise reception and/or transmission will be impaired. The receiving antenna as a part in the system is responsible of turning the electromagnetic waves into its original form (electrical signal in wire). Speaking of dipole antenna is speaking of omnidirectional antennas which radiates in all directions. Directed antennas are another category of antennas. The term directional antenna is used for antennas which radiates power in focused and specific direction. Directional antennas can be fixed in a specific location and directed towards the receiver (or transmitter) such as in microwave communications, or it can require rotation facilities as in radars. The ability of the antenna in focusing power in one direction more than other directions is a measure of quality of the antenna and it is often expressed by the terms gain, directivity, front to back ratio, half-power beam width HPBW, and many other factors and parameters of the antenna. When combining two or more elements to build an antenna with a specific defined distance and specific defined phase shifters, we can obtain the desired radiation pattern. A very common type of antennas is Yagi-Uda antenna uses this principle. It can be seen in most households to receive UHF and VHF TV signals. Yagi-Uda antenna uses the principle of radiation coupling, in which the feeding is to one element and other elements will be activated by it. The parabolic reflector dish is

the most common type of antennas when high gain is required. It has been used since the early of 1900s. It became very popular under the World War 11 in radar applications and in the present time it can be seen in almost household. The main advantage of the parabolic antenna is the large gain and directivity; however the main disadvantage is the big size dishes which are not easy to mount and have large windage. The principle is the same as the optical mirror reflector, when the source of beams is at the focus on the axis of the parabola, the reflected beam from the parabola will be parallel to the axis of the parabola. The horn antenna is very widely used in microwave applications since the early of 1900s. The name horn antenna comes from the appearance of this type of antennas. The flare of the horn antenna can be square, rectangular, cylindrical, or conical. Horn antennas are very easy fed with waveguide, but it can be fed also by a coaxial cable and a proper transition. Horn antennas are widely used as the active element of the parabolic reflector antenna where the horn is pointed towards the center of the parabolic antenna. The principle of how it works is very simple. If a waveguide is terminated it will radiate energy producing a broad radiated pattern. The Microstrip Patch Antenna is a single-layer design which consists generally of four parts (patch, ground plane, substrate, and the feeding part). Patch antenna can be classified as single - element resonant antenna. Once the frequency is given, everything (such as radiation pattern input impedance, etc.) is fixed. Because of the antenna is radiating from one side of the substrate, so it is easy to feed it from the other side (the ground plane), or from the side of the element. The most important thing to be considered is the maximum transfer of power (matching of the feed line with the input impedance of the antenna), this will be discussed later in the section of Impedance Matching. Many good designs have been discarded because of their bad feeding. The designer can build an antenna with good characteristics and good radiation parameter and high efficiency but when feeding is bad, the total efficiency could be reduced to a low 
level which makes the whole system to be rejected.

\section{II.ANALYSIS}

With the advent of adaptive materials, active antennas are now being developed that employ piezoelectric stacks or electrostatic actuation mainly for optical and far field pattern correction. The antennas proposed in this study will be utilized for beam scanning and shaping purposes where larger deflections are needed. Unlike the aperture antennas of old, these antennas are lightweight, flexible and can be designed to produce a "customer defined" far field radiation pattern. Recent theoretical and experimental studies have shown that by varying the surface of a reflector one can produce antennas that can achieve "customer defined" patterns while simultaneously adding the advantage of shape variation. Reflector aperture variation achieves most of the objectives of phased array technology while adding the ability to correct for surface distortions caused by weather, thermal gradients, and atmospheric effects. An added feature of these antennas, which is of great military benefit, is the ability to transmit to a large area for maximum ground coverage and then by varying the antenna shape transmit to a very small area for security reasons. Recent studies have shown that reflector surface adaptation can achieve performance characteristics on the order of phase array antennas without the complexity and cost. The work proposed in this study develops a class of antennas capable of variable directivity (beam steering) and power density (beam shaping). The actuation for these antennas is employed by either polyvinylidene fluoride film bonded to a metalized mylar skin or shape memory alloys embedded in a composite structure. Theoretical studies of flexible antennas have shown that cylindrical antennas can achieve a directivity variation (beam scanning) of over $10^{\circ}$ and an increase in ground coverage of over $40 \%$. Aperture efficiency: In general, the aperture of an antenna is not directly related to its physical size. However some types of antennas, for example parabolic dishes and horns, have a physical aperture (opening) which collects the radio waves. In these aperture antennas, the effective aperture $\mathrm{A}_{\text {eff }}$ must always be less than the area of the antenna's physical aperture $A_{\text {phys }}$, as can be seen from the definition above. An antenna's aperture efficiency, $e_{a}$ is defined as the ratio of these two areas:

$$
\text { ea }=\text { Aeff /Aphys }
$$

The aperture efficiency is a dimensionless

Parameter between 0 and 1.0 that measures how close the antenna comes to using all the radio power entering its physical aperture. If the antenna were perfectly efficient, all the radio power falling within its physical aperture would be converted to electrical power delivered to the load attached to its output terminals, so these two areas would be equal $A_{\text {eff }}=A_{\text {phys }}$ and the aperture efficiency would be 1.0. But all antennas have losses, such as power dissipated as heat in the resistance of its elements, nonuniform illumination by its feed, and radio waves scattered by structural supports and diffraction at the aperture edge, which reduce the power output. Aperture efficiencies of typical antennas vary from 0.35 to 0.70 but can range up to 0.90 .

Aperture and gain: The directivity of an antenna, its ability to direct radio waves in one direction or receive from a single direction, is measured by a parameter called its gain, which is the ratio of the power received by the antenna to the power that would be received by a hypothetical isotropic antenna, which receives power equally well from all directions.

$$
\mathrm{A}_{\mathrm{eff}}=\lambda^{2} / 4 \pi-------------2
$$

Where $\lambda$ is the wavelength of the radio waves. So the gain of any antenna is proportional to its aperture:

$$
G=\frac{A_{\text {eff }}}{\lambda^{2} / 4 \pi}=\frac{4 \pi A_{\text {phyr }} \epsilon_{e}}{\lambda^{2}}
$$

So antennas with large effective apertures are high gain antennas, which have small angular beam widths. Most of their power is radiated in a narrow beam in one direction, and little in other directions. As receiving antennas, they are most sensitive to radio waves coming from one direction, and are much less sensitive to waves coming from other directions. Although these terms can be used as a function of direction, when no direction is specified, the gain and aperture are understood to refer to the antenna's axis of maximum gain, or boresight. The performance and advantages of micro strip patch antennas such as low weight, low profile, and low cost made them the perfect choice for communication systems engineers. They have the capability to integrate with microwave circuits and therefore they are very well suited for applications such as cell devices, WLAN applications, navigation systems and many others. In this work; a compact rectangular patch antennas are designed and tested for GPS devices at $1.57542 \mathrm{GHz}$, and for a satellite TV signal at $11.843 \mathrm{GHz}$ and $11.919 \mathrm{GHz}$. The final part of this work has been concentrated on studying an array antenna with two and four elements. The antennas of the design examples of this work has been manufactured and tested in laboratory. This study will cover title of the study, significance of the study, aims and objectives of the study, research hypothesis and research design. This research has designed based upon descriptive study as it aims to identify and elaborate the objectives of work. This study combines both primary and secondary research methods. Thus, gathering and analyzing the data will be done on the basis of existing research. The current study is done to analyze the none super reactive antennas maximum directivity bounding and its Radiation pattern /radiation aperture.

\section{Radiation Pattern}

Radiation pattern is one of the important characteristic of an antenna as tells the spatial relative distribution of the electromagnetic wave generated by the antenna. The radiation pattern is a plot of the magnitude of the radiation field as a function of direction $(\theta, \eta)$. The radiation pattern is essentially a 3-D surface. In the field of antenna design the term radiation pattern (or antenna pattern or far-field pattern) refers to the directional (angular) dependence of the strength of the radio waves from the antenna or other source. Particularly in the fields of fiber optics, lasers, and integrated optics, the term radiation pattern, or near-field radiation pattern, may also be used as a synonym for the near-field pattern 
or Fresnel pattern.

Antenna Pattern: a graphical representation of the antenna radiation properties as a function of position.

\section{III.DESIRED APERTURE DISTRIBUTION}

For a more general case, the optimal aperture (in terms of both the size and the distribution) for the maximum penetration is investigated by two approaches: optimization and direct solving.

Optimization: Global optimization algorithms (such as Genetic Algorithm, Particle Swarm, Pattern Search, etc.) are widely used in electromagnetic problems. Genetic algorithm (GA) is applied to find the optimal aperture distribution in this work. To find the optimal aperture in this case, it is assumed that the aperture radius is fixed and only the distribution is optimized. For different aperture distributions of the same radius, $3 \mathrm{~dB}$ near-field beam radius is calculated at a range of distances. This range is selected around expected best focus distance from the case of uniform aperture. Then, the minimum $3 \mathrm{~dB}$ near-field beam radius in this range is selected as the fitness function which will be minimized by the means of GA. As for parameter to be tuned, low-order Fourier coefficients of current distribution are chosen. The purpose of this choice is to ensure the continuity and smoothness of resulting distribution. A sample of GA results, by using the above assumptions is presented here, for an aperture of radius equal to one wavelength (i.e. $\mathrm{a}=\lambda$ ) in free space. In this case a population of 20 genes is used and the algorithm converges after 51 generations. The amplitude and phase of resulting current distribution is shown in Fig. Near-field co-polar component produced by such distribution is demonstrated in Fig. at distances between $0.4 \lambda$ and $0.8 \lambda$, also the $3 \mathrm{~dB}$ near-field beam radius of this current distribution is plotted in Fig.

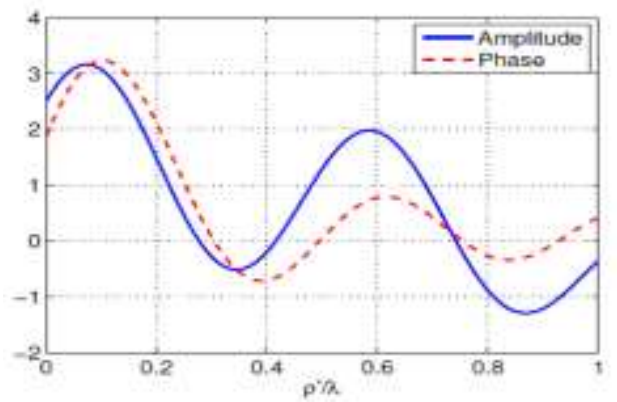

Fig. Amplitude and phase of current distribution from GA.

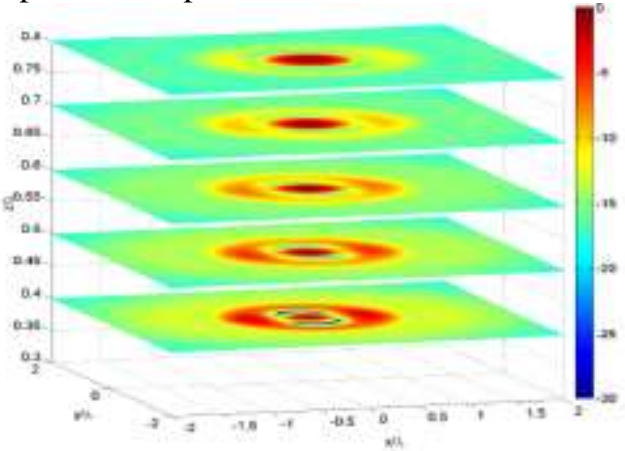

Fig. Near-field co-polar component of GA optimized aperture.

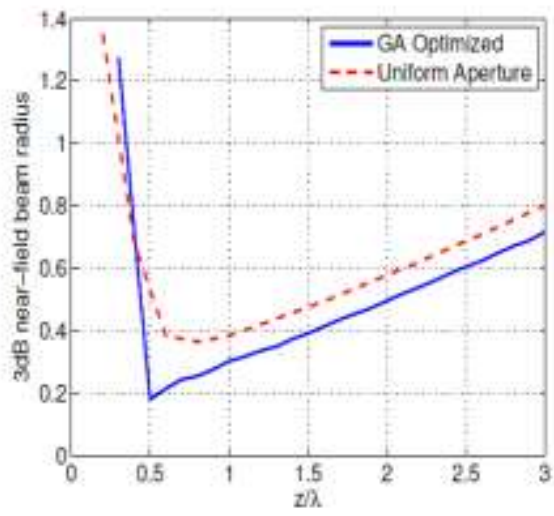

Fig. 3dB near-field beam radius of GA optimized aperture. Direct Solving: Due to the linear nature of the problem, it is also possible to solve directly for the aperture distribution to have a desired field distribution at one or more z-cuts in the space. In order to do this, following approach is adopted. First, a discrete aperture distribution $\operatorname{Jdis}(\rho)$ and a desired field Egoal are selected.

$$
J\left(\rho^{\prime}\right) \approx J_{\text {dis }}\left(\rho^{\prime}\right)=\sum_{n=1}^{N} J_{n} \psi_{n}\left(\rho^{\prime}\right)
$$

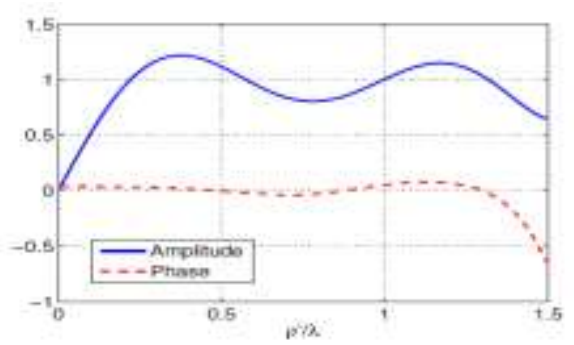

Fig. Calculated amplitude and phase of current distribution by using the direct solving method.

Types of Antenna Patterns -

Power Pattern - normalized power vs. spherical coordinate position.

Field Pattern - normalized $\mathrm{E}$ or $\mathrm{H}$ vs. spherical coordinate position.

\section{Antenna Field Types-}

Reactive field - The portion of the antenna field characterized by standing (stationary) waves which represent stored energy.

Radiation field -The portion of the antenna field characterized by radiating (propagating) waves which represent transmitted energy.

\section{Antenna Field Regions -}

Reactive Near Field Region - the region immediately surrounds the antenna where the reactive field (stored energy - standing waves) is dominant.

Near-Field (Fresnel) Region - the region between the reactive near field and the far-field where the radiation fields are dominant and the field distribution is dependent on the distance from the antenna.

Far-Field Region - the region farthest away from the antenna where the field distribution is essentially independent of the distance from the antenna (propagating waves). 


\section{Antenna Field Regions \& Definitions}

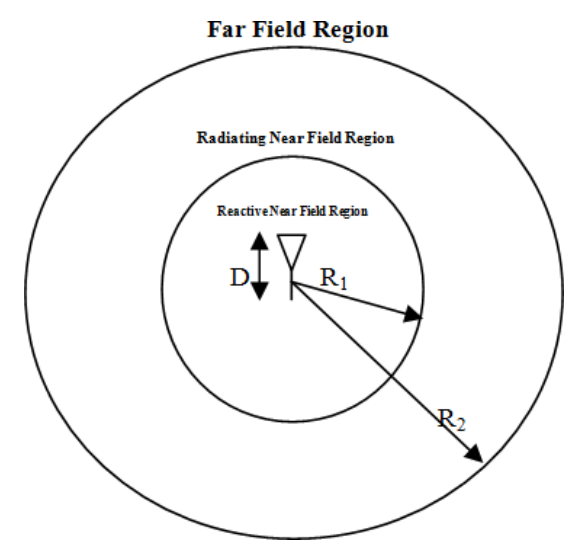

Figure: Antenna Field Regions

Isotropic Pattern - An antenna pattern defined by uniform radiation in all directions, produced by an isotropic radiator (point source, a non-physical antenna which is the only nondirectional antenna).

Directional Pattern - A pattern characterized by more efficient radiation in one direction than another (all physically realizable antennas are directional antennas).

Omnidirectional Pattern - A patter1n which is uniform in a given plane. Principal Plane Patterns - the E-plane and H-plane patterns of a linearly polarized antenna.

E-plane - The plane containing the electric field vector and the direction of maximum radiation.

H-plane - The plane containing the magnetic field vector and the direction of maximum radiation.

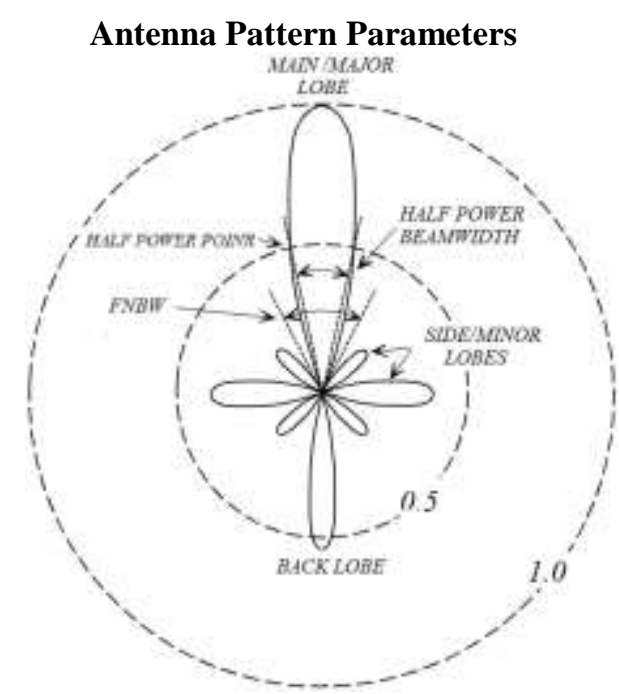

Figure: Antenna Pattern

Radiation Lobe -clear peak in the radiation intensity surrounded by regions of weaker radiation intensity.

Main Lobe (major lobe, main beam) - RL in the direction of maximum radiation.

Minor Lobe - any RL other than the main lobe.

Side Lobe - A RL in any direction other than the Direction(S) Of Intended Radiation.

Back Lobe - the RL opposite to the main lobe.
Half-Power Beam width - the angular width of the main beam at the half-power points.

First Null Beam width - angular width between the first nulls on either side of the main beam.

\section{IV.EFFECTS OF THE ANTENNAS}

On antenna Losses to obtain quantitative results for the effect of conduction losses on antenna performance an idealized model is again postulated. Intuitively one 'would expect the losses on a metal antenna to be smaller the more effectively the sphere is utilized. Therefore, for the ideal lossy antenna is postulated a spherical conductor of radius $\mathrm{R}$ excited by the magnetic sources on its surface. So long as the sphere is a good conductor the source will generate the desired field. If the conductivity of the sphere is poor, can be modified to allow for a field internal to the conductor.

\section{CONCLUSION}

In this paper, an optimized aperture distribution is investigated to achieve the maximum end fire directivity for a continuous line source with a uniform phase progression. The optimization method has the advantages of simplicity and efficiency, in which the aperture distribution is expanded into a set of cosine functions and the optimization, reduces to solving a constrained linear-least-squares problem. Based on the copious computed results from the optimization, an approximate closed-form expression is derived for the maximum directivity. The expression can serve as a bound for the end fire directivity for a line source with a uniform phase progression. It is found that the optimum directivity increases almost linearly with an increase of the normalized phase constant, but with a decrease in the radiated power. Nevertheless, the enhancement in the directivity is much higher than that in previously studied super directivity, under the same constraint in the ratio of reactive to radiated power. The current distribution does not oscillate widely as is does for super directivity, and is therefore more practical than for super directivity.

\section{REFERENCES}

[1] Y. Fujii, Y. Tsunemitsu, G. Yoshida, N. Goto, J. Hirokawa and M. Ando, "Partially Corporate Feed using E-plane Coupler in a Single-Layer Slotted Waveguide Array Antenna," IEICE Technical Report, AP2007- 150, 2008-1.

[2] M. Zhang, J. Hirokawa and M. Ando, "Fabrication of a Slotted Waveguide Array at $94 \mathrm{GHz}$ by Diffusion Bonding of Laminated Thin Plates," IEEE AP-S Int. Symp., paper\# 311.7 June 2009.

[3] L.A. Kurtz and J.S. Yee, "Second-order beams of twodimensional slot arravs," IRE Trans. Antennas Propag., Vol. 5, No. 4, pp. 356-362, Oct. 1957.

[4] S. Park, J. Hirokawa and M. Ando, "Simple analysis of a slot with a reflection-canceling post in a rectangular waveguide using only the axial uniform currents on the post surface," IEICE Trans. Commun., vol.E86-B, no. 8, pp. 2482-2487, Aug. 2003.

[5] S. R. Rengarajan, "Analysis of a center-inclined waveguide slot coupler," IEEE Trans. Microwave Theory Tech., Vol. 37, No. 5, pp. 884-339, May 1989.

[6] N.Goto, "A planar waveguide slot antenna of single layer structure," IEICE Technical Report, AP88-39, 1988.

[7] N.Goto, "A waveguide-fed printed antenna," IEICE Technical Report, AP89-3, 1989.

[8] S. Park, Y. Tsunemitsu, J. Hirokawa and M. Ando, "Center feed single layer slotted waveguide array," IEEE Trans. 
Antennas Propag., Vol. 54, No. 5, pp. 1474-1480, May 2006.

[9] Vengatesan K., and S. Selvarajan: Improved T-Cluster based scheme for combination gene scale expression data. International Conference on Radar, Communication and Computing (ICRCC), pp. 131-136. IEEE (2012).

[10] Kalaivanan M., and K. Vengatesan.: Recommendation system based on statistical analysis of ranking from user. International Conferenceon Information Communication and Embedded Systems (ICICES), pp.479- 484, IEEE, (2013).

[11] K. Vengatesan, S. Selvarajan: The performance Analysis of Microarray Data using Occurrence Clustering. International Journal of Mathematical Science and Engineering, Vol.3 (2) ,pp 69-75 (2014).

[12] Vengatesan K., Mahajan S.B., Sanjeevikumar P., Mangrule R., Kala V., Pragadeeswaran (2018) Performance Analysis of Gene Expression Data Using Mann-Whitney U Test. In: Konkani A., Bera R., Paul S. (Eds) Advances in Systems, Control and Automation. Lecture Notes in Electrical Engineering, vol 442. Springer, Singapore.

[13] MD. Javeed Ahammed, V.V.S.S.S.Chakravarthy , A. Swathi, International Conference on MicroElectronics,Electromagnetics and Telecommunications (ICMEET - Nov - 16) as "Performance of Firefly Algorithm for Null Positioning in Linear Arrays"

[14] MD. Javeed Ahammed, Dr. R.P. Singh, Dr. M. Satya Sai Ram, Published in IJARSE - Dec - 17, ISSN $2319-8354$ Journal as "A Non Superreactive Antennas Maximum Directivity Bounding and its Radiation Apperture"

[15] MD. Javeed Ahammed, Dr. R.P. Singh, Dr. M. Satya Sai Ram, Published in IJCRT - Mar - 18, ISSN 2320 - 2882 Journal as "A Non Superreactive Antennas Maximum Directivity Bounding and its Radiation Apperture"

[16] MD. Javeed Ahammed, Dr. R.P. Singh, Dr. M. Satya Sai Ram, Published in JARDCS(SCOPUS) - Mar - 18, ISSN 1943 - 023X Journal as "A NSA Maximum Directivity Bounding and its Radiation Apperture"

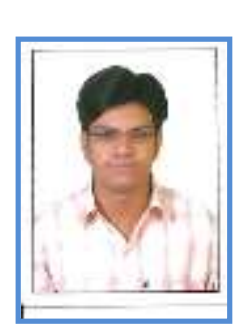

\section{AUTHORS}

MD. Javeed Ahammed ${ }^{1}$ is a Research Scholar in the Department of ECE at Sri Satya Sai University of Technology and Medical Sciences, Sehore, Madhya Pradesh. He has completed B.Tech and M.Tech in the ECE. He has been active in research for 2 years and published nearly 10 papers. His research interests Antennas, Communications, cellular Systems, and Digital Image Processing.

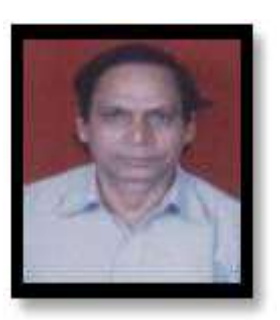

Dr. R.P. Singh ${ }^{2}$ has 40 years of teaching, research, and administrative experience as Professor. He has worked as Professor In-charge Academic and Chairman Admission Committee Dean (Academic) \& Dean (R/D) at Maulana Azad College of Technology /Maulana Azad National Institute of Technology, Bhopal. He has published 125 papers in National / International reputed and indexed Journals including SCI. He has worked as Secretary, Chairman, IETE, M.P. and C.G. and Council Member, IETE. He was first Counselor of IEEE student's chapter at Maulana Azad College of Technology, Bhopal. He has been member of Executive Committee, Institution of Engineers, and M.P. Circle. He was Chairman of Computer Society of India. Bhopal. He was member of Board of Studies, and Research Degree committee of many Universities. He has been Consulting Editor of Journal of Institution of Engineers and reviewer in many International/National Journals. Dr. Singh visited about 70 Institutions as an expert of National Board of Accreditation and about 35 Institutes as All India Council of Technical Education/ University Grants Commission. expert team for approval. Twenty candidates have completed their Ph.D. under his supervision and another six are registered in the area of Electronics/Communication system and related disciplines.

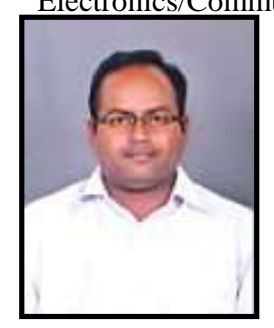

Dr. M. Satya Sai $\operatorname{Ram}^{3}$ has 15 years of teaching, research, and administrative experience as Professor. Obtained B.Tech degree and M.Tech degree from Nagarjuna University, Guntur. PhD from JNTUH, 2011. He has worked as Professor and HOD at Chalapathi Institute of Engineering and Technology, Chalapathi Nagar, Guntur, Andhra Pradesh, India. He actively involved in research and guiding for UG,PG AND PhD students in the area of ECE. He has taught a wide variety of courses for UG students and guided several projects. He has published 50 papers in National / International Conferences, Journals, Scopus and indexed Journals including SCI. He is a Life Member in Indian Society for Technical Education, International Association of Engineers \& Editorial Board Member Also. His research interests Antennas, Mobile/Cellular Systems, and Signal Processing and VLSI etc. 